\title{
Nutritional Management of Phenylketonuria
}

\author{
Erin L. MacLeod Denise M. Ney \\ Department of Nutritional Sciences, University of Wisconsin, Madison, Wisc., USA
}

\section{Key Words}

Phenylketonuria • Phenylalanine $\cdot$ Phenylalanine

hydroxylase $\cdot$ Long-chain polyunsaturated fatty acids •

Tetrahydrobiopterin · Amino acid

\begin{abstract}
Phenylketonuria (PKU) is caused by deficient activity of the enzyme phenylalanine hydroxylase, needed to convert the essential amino acid (AA) phenylalanine (phe) to tyrosine. In order to prevent neurological damage, lifelong adherence to a low-phe diet that is restricted in natural foods and requires ingestion of a phe-free AA formula to meet protein needs is required. The goal of nutritional management for those with PKU is to maintain plasma phe concentrations that support optimal growth, development, and mental functioning while providing a nutritionally complete diet. This paper reviews developing a lifelong dietary prescription for those with PKU, outcomes of nutritional management, compliance with the low-phe diet across the life cycle, and new options for nutritional management. An individualized dietary prescription is needed to meet nutrient requirements, and the adequacy of phe intake is monitored with assessment of blood phe levels. Elevated phe concentrations may occur due to illness, excessive or inadequate phe intake, or inade-
\end{abstract}

\section{KARGER}

Fax +4161306 1234

E-Mail karger@karger.ch

www.karger.com (c) 2010 Nestec Ltd., Vevey/S. Karger AG, Basel

Accessible online at: www.karger.com/ane quate intake of AA formula. Although normal growth and development occurs with adherence to the low-phe diet, it is important to monitor vitamin, mineral and essential fatty acid status, especially in those who do not consume sufficient AA formula. Given the growing population of adults with PKU, further research is needed to understand the risks for developing osteoporosis and cardiovascular disease. There are promising new options to liberalize the diet and improve metabolic control such as tetrahydrobiopterin therapy or supplementation with large neutral AAs. Moreover, foods made with glycomacropeptide, an intact protein that contains minimal phe, improves the PKU diet by offering a palatable alternative to AA formula. In summary, continued efforts are needed to overcome the biggest challenge to living with PKU - lifelong adherence to the low-phe diet.

Copyright $\odot 2010$ Nestec Ltd., Vevey/S. Karger AG, Basel

\section{Introduction}

Phenylketonuria (PKU) is an inborn error of amino acid (AA) metabolism caused by deficient activity of the enzyme phenylalanine hydroxylase (PAH), which is needed to convert the essential AA phenylalanine (phe) to tyrosine [1]. Nutritional management in the form of 
Table 1. Recommended guidelines for blood phe levels across Europe and the US

\begin{tabular}{lcccc}
\hline \multirow{2}{*}{ Age } & \multicolumn{4}{l}{ Blood phe level, mM } \\
\cline { 2 - 5 } & UK [5] & Germany [9] & France [8] & US [4] \\
\hline Birth $^{1}$ & $>400$ & $>600$ & $>600$ & $>600$ \\
Infant to 10 years & $120-360$ & $40-240$ & $120-360$ & $120-360$ \\
$10-12$ years & $<480$ & $<600$ & $<900$ & $120-360$ \\
$12-20$ years & $<480$ & $<600$ & $<900$ & $120-600$ \\
$>20$ years & $<700$ & $<1,200$ & $<900$ & $120-900$ \\
Pregnancy & & & & $120-360$ \\
\hline
\end{tabular}

${ }^{1}$ Phe concentration after newborn screening when countries initiate a low-phe diet. a low-phe diet is essential to achieve metabolic control of phe levels and allow normal development of the brain in those with PKU. Horst Bickel's group working at the Birmingham Children's Hospital in the UK in 1953 first reported the effectiveness of a low-phe diet in reducing phe concentrations and improving behavior in a child with PKU $[2,3]$. The key development of a source of dietary protein that was free of phe was achieved by acid hydrolysis of casein followed by treatment with activated charcoal to remove phe. Today, the major source of dietary protein for those with PKU consists of mixtures of synthetic AA devoid of phe and a small amount of dietary protein from fruits and vegetables to provide phe.

Implementation of newborn screening programs for PKU in the 1960s and initiation of a low-phe diet soon after birth has resulted in a worldwide population of approximately 50,000 individuals with PKU with cognitive ability in the normal range. The biggest challenge to living with PKU is the need for lifelong adherence to the highly restrictive low-phe diet supplemented with an AA formula $[4,5]$. Well-controlled studies show significant differences in performance and intelligence in children and neurologic function in adults who discontinue the low-phe diet compared with those who remain on diet [6]. Moreover, infants born to women with poorly controlled PKU are at risk for congenital malformations $[4,7]$. Thus, a low-phe diet remains the cornerstone for lifelong treatment of PKU.

The goal of nutritional management for those with PKU is to maintain plasma phe concentrations that support optimal growth, development, and mental functioning while providing a nutritionally complete diet. There are differences in opinion throughout the world regarding the recommended targets for plasma phe concentra- tions to optimize development and mental functioning for those with PKU, as summarized in table $1[4,5,8,9]$. There is consensus among European countries and the US that any newborn infant with a plasma phe concentration of $>400-600 \mu \mathrm{M}$ should be started on a low-phe diet as soon as possible, which is easily achieved in infancy and compatible with limited breastfeeding $[4,5]$. The low-phe PKU diet (in classical PKU, $<500 \mathrm{mg}$ phe per day [10]) requires two interrelated dietary modifications to achieve metabolic control of plasma phe concentrations. First, natural foods are severely restricted to limit protein intake yet provide adequate amounts of phe, an essential AA. This generally includes elimination of all sources of animal protein, legumes and nuts, as well as limited intake of bread, pasta, rice and some vegetables. Low-protein bread and pasta products made from starch are used to provide needed energy and to increase variety. Second, consumption of an AA-based, phe-free formula (600$900 \mathrm{ml}$ per day) or AA medical food is needed to provide adequate protein, vitamins, minerals and energy due to the restriction in natural foods.

This chapter will highlight developing a dietary prescription for those with PKU $[6,10]$, outcomes of nutritional management, compliance with the low-phe diet across the life cycle, and new options for the nutritional management of PKU.

\section{Developing and Assessing the Low-Phe Dietary Prescription}

Nutritional management of PKU is best accomplished by an experienced team of healthcare professionals including a physician, psychologist, metabolic dietitian, nurse and genetic counselor [11]. An individualized di- 
Table 2. Guidelines of daily protein, energy and phe intake for individuals with PKU

\begin{tabular}{|c|c|c|c|c|c|}
\hline \multirow[t]{2}{*}{ Age } & \multirow{2}{*}{$\begin{array}{l}\text { Protein requirement }{ }^{1} \\
\mathrm{~g} / \mathrm{kg}\end{array}$} & \multirow{2}{*}{$\begin{array}{l}\text { Minimum phe } \\
\text { requirement } \\
\mathrm{mg} / \mathrm{kg}\end{array}$} & \multirow{2}{*}{$\begin{array}{l}\text { Range of phe } \\
\text { intake } \\
\text { mg/day [10] }\end{array}$} & \multicolumn{2}{|l|}{ Energy [10] } \\
\hline & & & & $\mathrm{kcal} / \mathrm{kg} /$ day & kcal/day \\
\hline 0-6 months & $3-3.5$ & $20-70[10]$ & - & $95-145$ & \\
\hline $7-12$ months & $2.5-3(1.31)$ & $10-35$ [10] & - & $80-135$ & \\
\hline $1-3$ years & $2-3(1.02)$ & NA & $200-400$ & & $900-1,800$ \\
\hline $4-6$ years & $2(0.87)$ & $13-20[20]$ & $210-450$ & & $1,300-2,300$ \\
\hline $7-10$ years & $2(0.92)$ & $13-20[20]$ & $220-500$ & & $1,650-3,300$ \\
\hline \multicolumn{6}{|l|}{ Males } \\
\hline $11-14$ years & $2(0.90)$ & NA & $225-900$ & & $2,000-3,700$ \\
\hline $15-18$ years & $2(0.87)$ & NA & $295-1,100$ & & $2,100-3,900$ \\
\hline$\geq 19$ years & NA $(0.84)$ & $4.6-13.6$ [19] & $290-1,200$ & & $2,000-3,300$ \\
\hline \multicolumn{6}{|l|}{ Females } \\
\hline $11-14$ years & $2(0.89)$ & NA & $250-750$ & & $1,500-3,000$ \\
\hline $15-18$ years & $2(0.84)$ & NA & $230-700$ & & $1,200-3,000$ \\
\hline$\geq 19$ years & NA $(0.84)$ & $4.6-13.6$ [19] & $220-700$ & & $1,400-2,500$ \\
\hline
\end{tabular}

etary prescription is developed by initially assessing the age-specific requirements for protein and energy using established guidelines as shown in table 2.

Efficient utilization of AAs for synthesis of body proteins is influenced by many factors including the rate of protein digestion and absorption of AA into blood, presence of all essential or indispensable AA at the same time, and adequate intake of energy and total dietary nitrogen to support the high metabolic cost of protein synthesis. Protein requirements are thought to increase when the majority of protein is provided by an elemental AA-based diet compared to intact protein, due to rapid absorption of AA resulting in reduced utilization for protein synthesis $[12,13]$. The United Kingdom Medical Research Council Working Party on PKU recommend a total protein intake of at least $3 \mathrm{~g} / \mathrm{kg}$ body weight per day for children under 2 years of age and $2 \mathrm{~g} / \mathrm{kg}$ body weight per day for children over 2 years of age [5]. These amounts exceed recommendations for protein intake in the non-PKU population by approximately $30 \%$. However, evidence suggests that adults with PKU (excluding pregnancy) do not have increased protein requirements when the majority of dietary protein is provided by AA [14].

\section{Phe and Tyrosine}

Phe is essential in the diet for protein synthesis and must be provided in an amount that supports tissue repair, growth during childhood and protein turnover in adulthood while keeping plasma phe concentrations within recommended levels (table 1). Minimum phe requirements are not different for those with PKU than for individuals with normal PAH activity [15]. Current phe requirements reflect the combination of phe and tyrosine intake and have increased from $14 \mathrm{mg} / \mathrm{kg}$ per day to $25-27 \mathrm{mg} / \mathrm{kg}$ per day in adults $[16,17]$. The requirement for phe alone is difficult to interpret because of the wide reported range for conversion of phe to tyrosine in those with normal PAH activity $[1,18]$. The most accurate studies use stable isotope methodology to examine phe requirements in the presence of adequate tyrosine. Using this method and breakpoint analysis, Zello et al. [19] found the mean phe requirement for adults to be 9.1 $\mathrm{mg} / \mathrm{kg}$ and Courtney-Martin et al. [20] found a mean phe requirement of $14 \mathrm{mg} / \mathrm{kg}$ in children with PKU. Table 2 reflects these findings. Although there is a minimum phe requirement, the amount of phe tolerated in the diet must be assessed individually as specific gene mutations in $\mathrm{PAH}$ and tetrahydrobiopterin $\left(\mathrm{BH}_{4}\right)$ synthesis and recycling will affect the ability to convert phe 
to tyrosine and thus the dietary phe allowance for an individual with PKU. Table 2 provides information on phe requirements and typical ranges of phe tolerance across the life cycle as a guideline for developing an individualized dietary prescription for phe. The phe prescription for obese and overweight patients should be based on ideal body weight for height and age because phe needs are proportional to protein synthesis, which reflects lean body mass [21].

Tyrosine is an essential AA for those with PKU given the inability to normally hydroxylate phe to tyrosine. Tyrosine is a precursor for thyroxine, catecholamines and melanin, and plasma levels should be monitored in parallel with phe as many patients will show low tyrosine levels. However, tyrosine supplementation alone does not correct the PKU phenotype [1]. Although tyrosine is provided in large quantities in AA formulas, it is very insoluble and individuals may not receive enough if they do not shake their formula before consumption [6].

In order to promote protein synthesis and provide variety within a very restrictive diet, it is best to allow the maximum amount of natural dietary protein from breast milk or infant formula in infancy, and later in life from fruits, vegetables and low protein products. However, it is unusual for the diet of an individual with classical PKU to contain more than $25 \%$ of protein from natural foods (often only 5-10 g per day) given that protein in natural foods contains $2-9 \%$ phe by weight.

\section{Monitoring Phe Concentrations}

Frequent monitoring of plasma concentrations of phe and tyrosine in relation to phe intake, often provided by a 3-day record of food intake preceding blood sampling, is needed to adjust the dietary prescription for phe. When the growth rate is at its peak, as in early infancy and during the prepubertal and pubertal growth spurts, phe needs will be high relative to body mass and will then decline as the growth rate slows, table 2 . The phe ingested should maintain the 2- to 4-hour postprandial plasma phe concentration within the recommended target range [6]. During infancy, weekly adjustments in the phe prescription may be needed based on growth and assessment of plasma phe concentrations. Subsequently, phe is assessed once or twice monthly $[4,22]$.

Elevation in plasma phe concentration may occur due to a variety of reasons. Excessive phe intake, especially in the school age child or adolescent who is struggling to comply with his/her diet, is often the cause. Conversely, inadequate intake of phe, protein or total energy relative to that needed to support a growth spurt may also in- crease plasma phe concentration due to protein catabolism and release of phe into blood. In women, changes in plasma phe levels may occur during the menstrual cycle with higher phe levels in the late luteal phase. Infectious disease or trauma induces protein catabolism and negative nitrogen balance resulting in elevated plasma phe concentrations in those with PKU. Immediate treatment to prevent catabolism and elevated plasma phe concentrations is essential. This is often accomplished by adding additional carbohydrate to oral rehydration solutions which are then sipped throughout the day to provide an energy source that is phe free. For those that cannot tolerate oral feeding for a prolonged period, enteral feeding of a low-phe AA formula to provide energy, electrolytes and essential AA may be needed.

\section{Outcomes of Nutritional Management}

Normal growth and development is the positive outcome noted with early diagnosis and treatment of infants with PKU with a nutritionally adequate, low-phe diet. Evaluation of IQ scores in individuals with PKU treated shortly after birth through 12 years of age who maintained plasma phe concentrations within recommended treatment ranges show a mean IQ score of $101 \pm 11$ [23]. Waisbren et al. [24] reported that during the first 18 years of life for each $100 \mu \mathrm{mol}$ increase in plasma phe concentration between 394 and $750 \mu \mathrm{M}$, there was a 1.3to 3.9-point decline in IQ score. Metabolic control of PKU through nutritional management is clearly effective in promoting intellectual development. Moreover, ongoing nutritional assessment over the life cycle is essential for optimal metabolic control of PKU and to insure optimal growth. If a patient is not consuming an adequate amount of AA formula distributed throughout the day, not only will plasma phe concentration be elevated due to protein catabolism, but growth failure may occur because formula provides the majority of energy, protein and other essential nutrients.

\section{Vitamin, Mineral and Essential Fatty Acid Status}

Whenever a diet is elemental in nature with the elimination of natural food sources, such as the AA-based, low-phe diet, there is a potential for nutrient deficiencies. There is some evidence that with an elemental diet, intake of vitamins and minerals must be increased to compensate for poor absorption and metabolism. Since the development of AA-based formulas and medical foods in the 1960s, there have been continual improve- 
ments in the nutrient composition to correct nutritional problems. In particular low plasma levels of carnitine [25], selenium and zinc $[26,27]$ have promoted increased supplementation. Some AA products are devoid of vitamin and mineral supplements to improve taste and acceptability with the assumption that separate vitamin and mineral supplements will be taken each day. Nutrient intake, specifically of vitamin $B_{6}, B_{12}[28]$, calcium, folate and iron [29] will be deficient if the AA formula and/or vitamin and mineral supplements are not consumed each day. Interestingly, even with adequate iron intake, studies have shown iron deficiency in those with PKU [30].

Although not a low-protein diet, the PKU diet tends to contain more carbohydrate and less total fat (20-25\% energy) compared to a typical omnivore diet which provides $30-35 \%$ energy from fat $[31,32]$. Moreover, the diet is devoid of meat, poultry, fish, nuts, and dairy foods which are the primary sources of long-chain polyunsaturated fatty acids (LC-PUFAs) such as arachidonic acid (20:4n6), eicosapentaenoic acid (20:5n3), and docosahexaenoic acid (DHA, 22:6n3). DHA is known to support normal brain and visual development, and some medical foods for PKU are currently supplemented with DHA. Plasma levels of the n- 6 series of fatty acids, including linoleic acid and arachidonic acid, are generally normal in PKU; however, levels of the n-3 series of fatty acids, including linolenic acid and DHA, are decreased in treated children with PKU compared to control subjects [33]. Treated adults with PKU show the same trend, although the degree of reduction in DHA is less severe compared with children [34]. The evidence suggests that the PKU diet may be limiting in linolenic acid, the precursor of DHA. In addition, endogenous synthesis of DHA from linolenic acid may be impaired in PKU. Interestingly, a balanced dietary supplement of n-3 and n-6 LC-PUFAs maintained levels of arachidonic acid and eicosapentaenoic acid and restored DHA levels in plasma phospholipids, resulting in improved visual function in children with PKU [35]. In summary, blood lipid levels should be monitored, intake of vegetable oils rich in linolenic acid such as canola or soybean oil should be encouraged, and balanced supplements of n-3 and n-6 LC-PUFAs provided as needed for all individuals with PKU [34].

\section{Cardiovascular Disease}

As the first generation of PKU patients treated with synthetic AA formula for the majority of their lives reaches middle age, questions arise about PKU and the risk for cardiovascular disease. Several reports indicate lower plasma total cholesterol concentrations with normal HDL cholesterol concentrations in treated children and adults with PKU compared to control subjects without PKU $[1,33]$. Children with well-controlled PKU show significantly lower levels of atherogenic LDL cholesterol and apolipoprotein B and high levels of adiponectin, an adipose-tissue-derived hormone that prevents endothelial inflammation and early atherogenesis, compared to control subjects without PKU [32]. While these favorable parameters suggest protection from atherogenesis and cardiovascular disease, approximately one third of PKU subjects show total plasma homocysteine concentrations that exceed the 97th age centile, suggesting an increased risk for thrombosis, atherosclerosis and stroke [36]. Evidence suggests that deficient intake or altered metabolism of the $B$ vitamins, $B_{6}, B_{12}$ and folate that serve as cofactors for metabolism of homocysteine to methionine, contributes to elevated homocysteine levels in PKU $[32,36]$. In summary, it is unclear if those with PKU have an altered risk for cardiovascular disease because of their genetic profile or their unique diet that eliminates animal fat and provides the majority of nutrients from a synthetic AA formula.

\section{Osteopenia}

Long-term complications of low bone mass (i.e. osteopenia, osteoporosis and fractures) are becoming evident in the growing population of adolescents and adults with PKU. Studies of young adults with PKU found low peak bone mass in more than $40 \%$ of subjects $[37,38]$. The etiology of bone complications in PKU remains unclear and may reflect the PKU genotype and/or the composition of the AA-based diet, in particular reduced absorption and utilization of calcium, phosphorus and vitamin D. For genotype effects, the bone cells (osteoblasts and osteoclasts) or other tissues involved in calcium uptake and vitamin D metabolism (e.g. kidney and intestines) may be sensitive to elevated phe levels, just as brain cells are. In support of this, evidence from the PKU mouse model $\left(\mathrm{PAH}^{\mathrm{enu}}\right)$ suggests that increased circulating phe concentrations adversely affect bone development [39]. The current diet may provide an excess acid load [31] and a less than optimal supply of AA needed to maintain adequate protein synthesis and collagen turnover for formation of bone matrix [40]. Studies in humans with PKU are needed to investigate the complication of low bone mass in a longitudinal manner with careful assessment of dietary intake.
62

Ann Nestlé [Engl] 2010;68:58-69
MacLeod/Ney 


\section{Compliance across the Life Cycle}

The goal throughout the life cycle is continuous, uninterrupted adherence to the low-phe diet because once discontinued, it is extremely difficult to reintroduce. Compliance with the PKU diet and achievement of metabolic control is easily accomplished in infancy and early childhood before children start school. Not surprisingly, compliance becomes a significant problem during adolescence. The reasons for poor dietary compliance are multifactorial, and the ramifications range from frequent headaches to the devastating effects of spastic quadriparesis and maternal PKU.

\section{Early Childhood}

In infancy and early childhood, phe-free AA formula provides most of the protein, energy and nutrient needs for growth. Initially, dietary phe requirements are met by small amounts of breast milk or infant formula. As feeding skills develop, phe requirements are met through small amounts of rice cereal and puréed fruits and vegetables. With continued development of feeding skills, toddlers with PKU meet their phe requirements from low-protein table foods. Extensive lists and pocket guides for the phe content of different foods have been published to assist families in monitoring phe intake [41]. Phe tolerance is individualized for each child according to their genotype and function of the PAH enzyme and is clinically determined by frequent monitoring of blood phe concentrations and dietary phe intake. During the toddler and early childhood years, the phe prescription changes rapidly as children grow and protein needs change (table 2). Although the PKU diet in early childhood requires a large intake of AA formula to meet protein needs (2-3 g protein $/ \mathrm{kg})$ and strict control of phe intake, compliance is good, presumably due to high parental control and low peer pressure.

\section{School-Aged Children and Adolescence}

Compliance with the PKU diet becomes more difficult as children grow older and start school, make their own food choices and develop taste preferences which are often similar to those without PKU [42]. Changes in compliance are usually related to consumption of the AA-based formula. Children and adolescents may transition from formula 3 or 4 times a day to only once or twice a day limiting the availability of AA, at a time of development when protein synthesis is essential for growth. Frequent consumption of AA formula throughout the day results in stable plasma phe concentrations and higher phe tolerance as protein retention and synthesis improve [21, 43]. Consuming an AA formula at school is the most challenging aspect of compliance as AA formula looks different and has a distinctive poor odor and taste, in spite of effor ts to improve formulations and packaging $[44,45]$. Most children refuse to drink the formula at school, thus are without a good source of protein for $8 \mathrm{~h}$ resulting in catabolism of body protein and release of the phe into blood. Moreover, significant hunger may cause them to consume too much phe from prescribed or forbidden foods. Both of these factors lead to elevated blood phe concentrations with increased age [46], which negatively affects IQ [24].

Since PKU requires lifelong diet therapy, it is paramount that children and adolescents learn the importance of dietary adherence. Children should progressively assume responsibility for diet management starting at an early age to establish healthy habits and prevent diet discontinuation. In order to improve dietary compliance, innovative programs such as a camp for individuals with PKU attempted to provide an outlet for peer support and diet education, and although effective in the short term, did not result in improved compliance 1 year later [47]. Clearly, dietary education and knowledge are not the only factors in adolescent compliance, but continued support and reinforcement are needed particularly considering that $50 \%$ of those $10-14$ years of age have blood phe concentrations above the recommended target, and this increases to $79 \%$ for those 15-19 years of age [46].

\section{Adulthood}

Unless the diet was discontinued in adolescence, the practices learned in childhood will likely continue throughout adulthood. The individual who has stopped the diet as a teenager, will often attempt to reinstitute the diet as an adult. However, it is extremely difficult to reestablish the low-phe diet as shown by one survey which reported that $31 \%$ of patients who had attempted to reintroduce the diet were again off diet after 10 months [48] noting the primary mediator as decreased motivation. Decreased motivation to stay on diet may be related to the absence of immediate effects of noncompliance, unlike a condition such as diabetes. An individual who began the PKU diet at birth and is now noncompliant may exhibit increased irritability, difficulty concentrating, or headaches, and often it is family members who notice the effects before the individual with PKU does. Bik-Multanowski et al. [49] found that only 55\% of adults were able to achieve dietary compliance for 3 months after diet 
Fig. 1. Blood phe response to increased phe intake in 2 adults with PKU. Pattern of blood phe concentrations from 2 subjects with biweekly increases in dietary phe intake of $10-20 \%$. Both subjects were adult women with PKU in good control. Reassessment of phe tolerance included daily food records and blood phe monitoring every other day. Values are mean \pm SEM.

reintroduction, and only $19 \%$ were able to follow the diet for 9 months. However, those who returned to diet showed increases in quality of life measurements, consistent with previous findings [50].

Those adults who remain on diet often do not return to their metabolic clinic for regular checkups, assuming that their phe prescription as well as food and formula options are unchanged. Although PAH activity is likely determined by an individual's genotype, phe prescriptions will change throughout the life span as individuals gain weight. An innovative approach to reassess the PKU diet for adults with PKU [21] demonstrated that 7 of 8 adults were able to significantly increase their phe intake, and in some cases double it, without a significant increase in plasma phe concentrations. The blood phe concentrations during diet reassessment for two subjects who increased their phe prescription to reflect adult phe requirements are shown in figure 1.

\section{Maternal PKU}

The consequences of poor dietary compliance are devastating when a woman with PKU becomes pregnant, termed maternal PKU. Exposure of the fetus to high maternal phe concentrations results in microcephaly, congenital heart defects, intrauterine growth retardation and learning disabilities [7]. The maternal to fetal concentration gradient results in phe levels 1.5 to 2 times greater in the fetus than in the maternal plasma. Therefore, it is recommended that women achieve plasma phe concentrations of 120 to $360 \mu \mathrm{M}$ [4] prior to pregnancy as critical

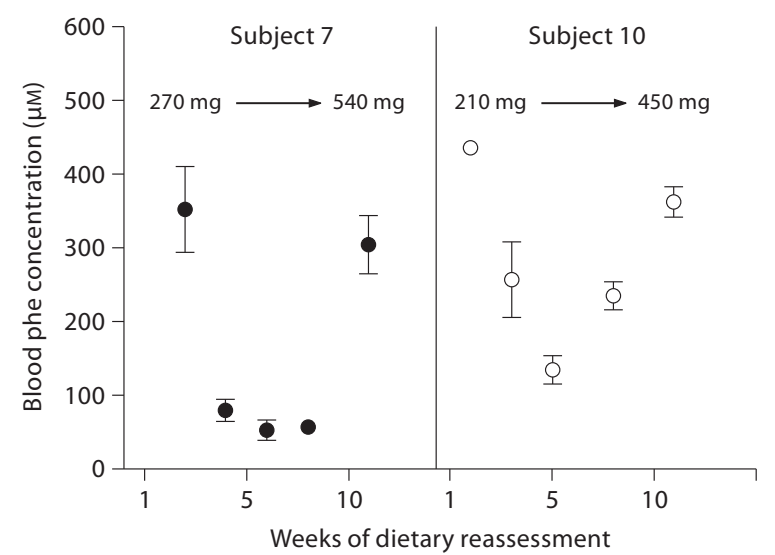

development of the fetal central nervous system and heart occurs between 5 and 8 weeks' gestation. Studies from the Maternal PKU Collaborative Study showed that normal intellectual development was achieved in children born to women with PKU if maternal blood phe levels were below $600 \mu \mathrm{M}$ by 10 weeks' gestation [51]. This is extremely difficult to achieve even with added support during pregnancy [52], and the biggest challenge, but key to achieving metabolic control, is sufficient consumption of a phe-free AA formula. Moreover, given the increased requirements for protein, vitamins and minerals during pregnancy, consumption of AA formula is essential. For women who are unable to consume the required amount of formula and achieve plasma phe recommendations, placement of a gastrostomy tube can be an effective method of formula delivery.

\section{New Options to Treat PKU}

Given the poor dietary compliance with the low-phe diet after early childhood, new approaches are needed to reduce phe concentrations in plasma and brain with potential to liberalize the diet by allowing for a greater daily phe allowance. Administration of $\mathrm{BH}_{4}$ and large neutral AAs (LNAAs) are two new approaches to therapy that have recently been introduced and shown to be effective in selected individuals with PKU. 


\section{Tetrahydrobiopterin}

$\mathrm{BH}_{4}$, a required cofactor for the $\mathrm{PAH}$ enzyme, has recently been approved as a treatment for individuals with PKU to enhance PAH activity and ultimately phe tolerance. Response to $\mathrm{BH}_{4}$ therapy has been defined as a $30 \%$ decrease in blood phe following treatment, and although response rates vary, a positive response appears to be most common in those with a mild form of PKU [53-55]. Children who responded to $\mathrm{BH}_{4}$ therapy are able to increase their daily phe prescription by approximately 500 $1,000 \mathrm{mg}$ phe [55] or from 18 to $40 \mathrm{mg}$ phe per kg body weight [56], allowing for the addition of 2-3 servings of typical bread and cereal products in place of the usual low protein products. Although increased phe tolerance has the potential to improve overall control of plasma phe concentrations, few individuals with PKU are able to discontinue the low-phe diet including the AA formula and therefore need continued follow-up and assessment. Protocols are available for determination of responsiveness to doses of $5-20 \mathrm{mg} / \mathrm{kg}$ per day of $\mathrm{BH}_{4}$ [57]. Singh et al. [58] outlined a protocol for dietary management of those with $\mathrm{PKU}$ who respond to $\mathrm{BH}_{4}$ which includes continued evaluation of plasma phe concentrations during a controlled phe challenge with milk or egg powder. The assistance of a metabolic dietitian is needed to educate individuals about appropriate adjustments of phe intake as well as ensuring adequate energy and micronutrient status with the potential for decreased consumption of AA formula. Few $\mathrm{BH}_{4}$ responsiveness studies have controlled for overall energy and protein intake but are based on the individual's recall of their usual diet which may not be reliable. Given recent evidence that dietary counseling resulted in an increase in phe tolerance in adults with PKU who did not receive a $\mathrm{BH}_{4}$ trial [21], individuals may benefit from a thorough dietary assessment of phe tolerance prior to a $\mathrm{BH}_{4}$ trial. Although the high cost of $\mathrm{BH}_{4}$ therapy is a challenge, the potential for $\mathrm{BH}_{4}$ to improve overall metabolic control and health for those who respond is promising.

\section{LNAA Supplementation}

Phe competes with other LNAAs (including arginine, histidine, isoleucine, leucine, lysine, methionine, threonine, tryptophan, tyrosine and valine) for specific carrier proteins that transport LNAAs across the intestinal mucosa into the blood and across the blood-brain barrier into the brain [59]. The ability of LNAAs to compete for transporters is a function of the carrier protein's affinity for each AA. The carrier protein responsible for LNAA transport into the brain has a high affinity (low $\mathrm{Km}$ ) for phe, and this in combination with high plasma concentrations of phe relative to other AA unfortunately assures ample transport of phe into brain. The concentration of phe in brain is the best correlate of mental impairment in individuals with PKU. For example, there are intellectually normal persons with PKU who have never been treated with a low-phe diet and show elevated plasma phe concentrations but low levels of phe in the brain due to reduced transport [60]. Supplementation with LNAAs reduces influx of phe into the brain based on quantitative magnetic resonance spectroscopy consistent with competitive inhibition of phe transport due to increased plasma levels of other LNAAs [61]. Likewise, individuals with PKU given daily supplements of LNAAs (250-500 mg/ $\mathrm{kg}$ ) show significant (25-39\%) decreases in the concentration of phe in plasma consistent with competitive inhibition of phe absorption from the gut $[62,63]$. LNAA supplementation is most effective in lowering plasma phe concentrations in individuals who have difficulty complying with diet and show elevated phe levels, often $>1,000 \mu \mathrm{M}[62,63]$. For individuals already complying with diet and taking AA formula, additional supplementation with LNAAs is of limited value [62]. The ability to measure brain phe concentration with magnetic resonance spectroscopy and show a correlation with plasma phe concentration, although potentially very useful, has proved challenging especially when the blood phe concentration is below $1,200 \mu \mathrm{M}$. Interestingly, Schindeler et al. [62] demonstrated that LNAA supplementation had a specific effect of improving executive functions particularly in verbal generativity and cognitive flexibility in subjects who showed a decrease from 1,180 to $958 \mu \mathrm{M}$ in plasma phe concentration. In summary, LNAA supplementation is not a substitute for food but, for those individuals unable to comply with the low-phe diet, including cognitively impaired adults who were born prior to newborn screening, LNAAs may offer a cost-effective option to improve metabolic control.

\section{Glycomacropeptide Provides a New Paradigm for the PKU Diet}

With the exception of successful gene or enzyme replacement therapy, new therapies for PKU are unlikely to allow those with classical PKU to eat a completely normal diet. Thus, new dietary options are clearly needed to improve the acceptability of the low-phe diet to achieve lifelong metabolic control of PKU. Glycomacropeptide (GMP), a natural protein found in sweet cheese whey, is 
Fig. 2. Concentration of total AAs and blood urea nitrogen in postprandial plasma with ingestion of the GMP or the AA diet. Plasma was obtained $2.5 \mathrm{~h}$ after eating breakfast. Total plasma AA indicates the sum of all AAs measured in plasma. Values are means \pm SEM. Total plasma AAs increased and blood urea nitrogen decreased with ingestion of the GMP diet when compared with day 4 of the AA diet. ${ }^{*} \mathrm{p}<0.05$, significantly different from the AA diet on day 4 (paired t test, pairing on subject). Adapted from Van Calcar et al. [31].

uniquely suited to the PKU diet because it is the only known dietary protein that contains no phe in its pure form. Moreover, GMP contains significant amounts of the nontoxic LNAA, isoleucine, threonine and valine. GMP must be supplemented with 5 limiting AA (arginine, histidine, leucine, tryptophan, and tyrosine) to serve as a complete source of protein for those with PKU. Studies in the PKU mouse model demonstrate that GMP, supplemented with limiting AA, provides an adequate source of protein that supports normal growth and improves metabolic control by significantly reducing concentrations of phe in plasma and brain compared to an AA diet [64].

Unlike synthetic AA, GMP has functional properties suitable for making foods, including good heat stability and solubility in acid [65]. Highly acceptable low-phe, high-protein foods and beverages that provide 5-15 $\mathrm{g}$ of protein and only $15-25 \mathrm{mg}$ phe per serving can be made with GMP to provide an alternative to AA formula for those with PKU with a daily allowance of $\geq 300 \mathrm{mg}$ phe. These GMP foods include: beverages, either milkshakelike or clear, fruit flavored; pudding; puffed cereal; crackers; salad dressings, and a snack bar. At the conclusion of a clinical study establishing short-term safety of GMP, 10 of 11 PKU subjects felt that these GMP foods were better tasting and added variety to the low-phe diet compared to AA formula [31].

The current AA-based low-phe diet provides approximately $80 \%$ of protein needs from synthetic AA and $20 \%$ from the intact protein found in fruits and vegetables [6]. In contrast, a GMP-based low-phe diet supplemented

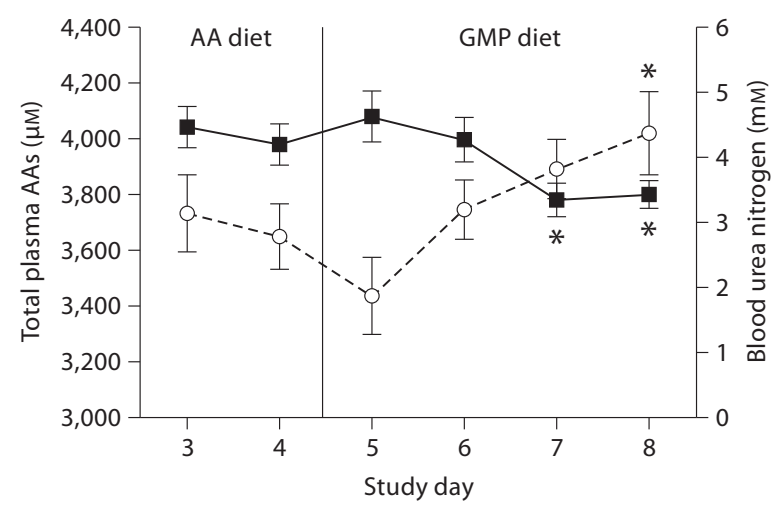

with limiting AA provides approximately $70 \%$ of protein needs from intact protein (found in GMP, fruits and vegetables) and only $30 \%$ of protein from synthetic AA needed to supplement the limiting AA in GMP. This represents a new paradigm to move the current PKU diet from synthetic AA to a more physiologic, normalized diet based on intact protein. Studies in humans using stable isotopes suggest that ingestion of intact protein compared with AA increases synthesis of somatic protein and decreases oxidation of AA due to slower absorption of AA from intact protein $[12,13,40]$. An inpatient metabolic study in PKU subjects [31] showed that ingestion of GMP sustained higher levels of AA in plasma and improved protein retention based on lower serum blood urea nitrogen and higher plasma insulin concentrations compared with ingestion of an AA-based diet (fig. 2). These data are consistent with the view that substitution of nutritionally complete foods made with GMP for AA formula will change the low-phe diet to be more physiologic and better support satiety $[66,67]$ and protein synthesis [31].

Clinical studies comparing a GMP diet with the usual AA-based diet in PKU subjects indicate that GMP shows promise to improve the nutritional management of PKU $[31,68]$. One subject who safely followed the GMP diet for 10 weeks at home, showed a significant (14\%) reduction in mean blood phe concentration compared with his usual AA diet [68]. Several explanations may account for the improvement in blood phe concentration with the GMP diet. First, because the subject enjoyed the GMP foods he was able to space them throughout the day, unlike his AA formula which he drank all at once. Distribution of AA 
formula throughout the day improves protein retention and reduces blood phe levels in those with PKU as discussed earlier. Second, GMP provides significant amounts of LNAAs, in particular threonine which has been shown to reduce plasma phe concentrations in those with PKU [69]. A greater intake of LNAAs from GMP may have reduced intestinal absorption of phe and contributed to lower blood phe levels. In summary, GMP provides a new paradigm for the PKU diet through the use of low-phe, high-protein, good-tasting foods made with the intact protein GMP, instead of synthetic AA.

\section{Conclusions}

Successful newborn screening programs to diagnose PKU and initiate management with a low-phe diet have resulted in a growing population of generally healthy, middle-aged adults with PKU. Compliance with the low-phe AA-based diet is very good in infancy and early childhood; however, many individuals with PKU become lax in their adherence to the strict diet as they age, resulting in elevated levels of phe and subsequent neuropsychological deterioration. The biggest challenge to living with PKU is lifelong adherence to a diet that allows only a small intake of natural foods and requires sufficient intake of AA formula to provide nutritional adequacy. New therapies which may help to liberalize the strict diet for some with PKU include $\mathrm{BH}_{4}$ drug therapy and LNAA supplementation. GMP shows promise to improve the taste, variety and acceptability of the low-phe diet, the cornerstone of metabolic control. As PKU evolves from a childhood diagnosis to an adult condition, there is a need for new guidelines to integrate nutritional, neuropsychological and psychosocial issues with target plasma phe concentrations to improve quality of life for adults with PKU [70]. Future needs to optimize health for those living with PKU include specialized clinics for the management of metabolic disorders in adults and continued research to improve understanding of how PKU affects the progression of chronic disorders such as cardiovascular disease and osteoporosis. The history of PKU is rich with accomplishments and the future for those living with $\mathrm{PKU}$ is promising.

\section{Acknowledgements}

The authors would like to thank Sally Gleason, MS, RD, for her invaluable input in preparation of the manuscript.

This study was supported by NIH grants R03-DK-071534, P30-HD-03352, 1UL1RR025011 from the Clinical and Translational Science Award Program of the National Center for Research Resources, and the College of Agricultural and Life Sciences.

\section{References}

1 Donlon J, Levy H, Scriver C: Hyperphenylalaninemia: Phenylalanine hydroxylase deficiency; in Scriver C, Beaudet A, Sly W, Valle $\mathrm{D}$ (eds): Metabolic and Molecular Basis of Inherited Disease. New York, McGraw-Hill, chapter 77, 2007.

-2 Bickel H, Gerrard J, Hickmans EM: Influence of phenylalanine intake on phenylketonuria. Lancet 1953;265:812-813.

3 Bickel H, Gerrard J, Hickmans EM: The influence of phenylalanine intake on the chemistry and behaviour of a phenyl-ketonuric child. Acta Paediatr 1954;43:64-77.

4 NIH: Phenylketonuria (PKU): screening and management. NIH Consensus Statement $2000 ; 17: 1-33$.

5 Recommendations on the dietary management of phenylketonuria. Report of Medical Research Council Working Party on Phenylketonuria. Arch Dis Child 1993;68:426-427.
6 Acosta PB, Matalon KM: Nutrition management of patients with inherited disorders of aromatic amino acid metabolism; in Acosta PB (ed): Nutrition Management of Patients with Inherited Metabolic Disorders. Boston, Jones and Bartlett Publishers, 2010, pp 119174.

7 Guttler F, Azen C, Guldberg P, et al: Relationship among genotype, biochemical phenotype, and cognitive performance in females with phenylalanine hydroxylase deficiency: report from the Maternal Phenylketonuria Collaborative Study. Pediatrics 1999;104:258-262.

-8 Abadie V, Berthelot J, Feillet F, et al: Management of phenylketonuria and hyperphenylalaninemia: the French guidelines (in French). Arch Pediatr 2005;12:594-601.

-9 Burgard P, Bremer HJ, Buhrdel P, et al: Rationale for the German recommendations for phenylalanine level control in phenylketonuria 1997. Eur J Pediatr 1999;158:46-54.
10 Acosta P, Yannicelli S: Protocol 1 - phenylketonuria (PKU); in The Ross Metabolic Formula System Nutrition Support Protocols, ed 4. Columbus, Ross Products Division/Abbot Laboratories, 2001.

11 Camfield CS, Joseph M, Hurley T, et al: Optimal management of phenylketonuria: a centralized expert team is more successful than a decentralized model of care. J Pediatr 2004; 145:53-57.

12 Metges CC, El-Khoury AE, Selvaraj AB, et al: Kinetics of L-[1-(13)C]leucine when ingested with free amino acids, unlabeled or intrinsically labeled casein. Am J Physiol Endocrinol Metab 2000;278:E1000-E1009.

13 Dangin M, Boirie Y, Garcia-Rodenas C, et al: The digestion rate of protein is an independent regulating factor of postprandial protein retention. Am J Physiol Endocrinol Metab 2001;280:E340-E348. 
-14 van Rijn M, Hoeksma M, Sauer P, Szczerbak B, Gross M, Reijngoud DJ, van Spronsen F: Protein metabolism in adult patients with phenylketonuria. Nutrition 2007;23:445453.

15 Kindt E, Halvorsen S: The need of essential amino acids in children. An evaluation based on the intake of phenylalanine, tyrosine, leucine, isoleucine, and valine in children with phenylketonuria, tyrosine amino transferase defect, and maple syrup urine disease. Am J Clin Nutr 1980;33:279-286.

16 IOM: Dietary Reference Intakes for Energy, Carbohydrates, Fiber, Fat, Protein and Amino Acids (Macronutrients). Washington, National Academy Press, 2002.

17 WHO: Protein and Amino Acid Requirements in Human Nutrition. Geneva, WHO, 2007.

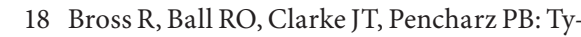
rosine requirements in children with classical PKU determined by indicator amino acid oxidation. Am J Physiol Endocrinol Metab 2000;278:E195-E201.

-19 Zello GA, Pencharz PB, Ball RO: Phenylalanine flux, oxidation, and conversion to tyrosine in humans studied with L-[1-13C]phenylalanine. Am J Physiol 1990;259:E835E843.

20 Courtney-Martin G, Bross R, Raffi M, et al: Phenylalanine requirement in children with classical PKU determined by indicator amino acid oxidation. Am J Physiol Endocrinol Metab 2002;283:E1249-E1256.

-21 MacLeod EL, Gleason ST, van Calcar SC, Ney DM: Reassessment of phenylalanine tolerance in adults with phenylketonuria is needed as body mass changes. Mol Genet Metab 2009;98:331-337.

-22 van Spronsen FJ, Ahring KK, Gizewska M: PKU - what is daily practice in various centres in Europe? Data from a questionnaire by the scientific advisory committee of the European Society of Phenylketonuria and $\mathrm{Al}$ lied Disorders. J Inherit Metab Dis 2009;32: 58-64.

23 Azen CG, Koch R, Friedman EG, et al: Intellectual development in 12-year-old children treated for phenylketonuria. Am J Dis Child 1991;145:35-39.

24 Waisbren SE, Noel K, Fahrbach K, et al: Phenylalanine blood levels and clinical outcomes in phenylketonuria: a systematic literature review and meta-analysis. Mol Genet Metab 2007;92:63-70.

25 Weigel C, Kiener C, Meier N, et al: Carnitine status in early-treated children, adolescents and young adults with phenylketonuria on low phenylalanine diets. Ann Nutr Metab 2008;53:91-95.

26 Barretto JR, Silva LR, Leite ME, et al: Poor zinc and selenium status in phenylketonuric children and adolescents in Brazil. Nutr Res 2008;28:208-211.
27 van Bakel MM, Printzen G, Wermuth B, Wiesmann UN: Antioxidant and thyroid hormone status in selenium-deficient phenylketonuric and hyperphenylalaninemic patients. Am J Clin Nutr 2000;72:976-981.

28 Schulz B, Bremer HJ: Nutrient intake and food consumption of adolescents and young adults with phenylketonuria. Acta Paediatr 1995;84:743-748.

29 Hvas AM, Nexo E, Nielsen JB: Vitamin $B_{12}$ and vitamin $\mathrm{B}_{6}$ supplementation is needed among adults with phenylketonuria (PKU). J Inherit Metab Dis 2006;29:47-53.

30 Acosta PB, Yannicelli S, Singh RH, et al: Iron status of children with phenylketonuria undergoing nutrition therapy assessed by transferrin receptors. Genet Med 2004;6:96101.

- 31 Van Calcar SC, Macleod EL, Gleason ST, et al: Improved nutritional management of phenylketonuria by using a diet containing glycomacropeptide compared with amino acids. Am J Clin Nutr 2009;89:1068-1077.

- 32 Schulpis KH, Papassotiriou I, Tsakiris S, et al: Increased plasma adiponectin concentrations in poorly controlled patients with phenylketonuria normalize with a strict diet: evidence for catecholamine-mediated adiponectin regulation and a complex effect of phenylketonuria diet on atherogenesis risk factors. Metabolism 2005;54:1350-1355.

33 Agostoni C, Scaglioni S, Bonvissuto M, et al: Biochemical effects of supplemented longchain polyunsaturated fatty acids in hyperphenylalaninemia. Prostaglandins Leukot Essent Fatty Acids 2001;64:111-115.

34 Moseley K, Koch R, Moser AB: Lipid status and long-chain polyunsaturated fatty acid concentrations in adults and adolescents with phenylketonuria on phenylalanine-restricted diet. J Inherit Metab Dis 2002;25: 56-64.

35 Agostoni C, Massetto N, Biasucci G, et al: Effects of long-chain polyunsaturated fatty acid supplementation on fatty acid status and visual function in treated children with hyperphenylalaninemia. J Pediatr 2000;137: 504-509.

36 Huemer M, Huemer C, Moslinger D, et al: Growth and body composition in children with classical phenylketonuria: results in 34 patients and review of the literature. J Inherit Metab Dis 2007;30:694-699.

37 Perez-Duenas B, Cambra FJ, Vilaseca MA, et al: New approach to osteopenia in phenylketonuric patients. Acta Paediatr 2002;91:899904.

38 Zeman J, Bayer M, Stepan J: Bone mineral density in patients with phenylketonuria. Acta Paediatr 1999;88:1348-1351

39 Yannicelli S, Medeiros DM: Elevated plasma phenylalanine concentrations may adversely affect bone status of phenylketonuric mice. J Inherit Metab Dis 2002;25:347-361.
40 Deglaire A, Fromentin C, Fouillet H, et al: Hydrolyzed dietary casein as compared with the intact protein reduces postprandial peripheral, but not whole-body, uptake of nitrogen in humans. Am J Clin Nutr 2009;90: 1011-1022.

41 Schuett V: Low Protein Food List for PKU, ed 2, Seattle, National PKU News, 2002.

42 Owada M, Aoki K, Kitagawa T: Taste preferences and feeding behaviour in children with phenylketonuria on a semisynthetic diet. Eur J Pediatr 2000;159:846-850.

43 MacDonald A, Rylance G, Hall SK, et al: Factors affecting the variation in plasma phenylalanine in patients with phenylketonuria on diet. Arch Dis Child 1996;74:412-417.

-44 MacDonald A, Lilburn M, Davies P, et al 'Ready to drink' protein substitute is easier is for people with phenylketonuria. J Inherit Metab Dis 2006;29:526-531.

45 Prince AP, McMurray MP, Buist NR: Treatment products and approaches for phenylketonuria: improved palatability and flexibility demonstrate safety, efficacy and acceptance in US clinical trials. J Inherit Metab Dis 1997; 20:486-498.

46 Walter JH, White FJ, Hall SK, et al: How practical are recommendations for dietary control in phenylketonuria? Lancet 2002. 360:55-57.

47 Singh RH, Kable JA, Guerrero NV, et al: Impact of a camp experience on phenylalanine levels, knowledge, attitudes, and health beliefs relevant to nutrition management of phenylketonuria in adolescent girls. J Am Diet Assoc 2000;100:797-803.

48 Schuett VE, Brown ES, Michals K: Reinstitution of diet therapy in PKU patients from twenty-two US clinics. Am J Public Health 1985;75:39-42.

49 Bik-Multanowski M, Didycz B, Mozrzymas $\mathrm{R}$, et al: Quality of life in noncompliant adults with phenylketonuria after resumption of the diet. J Inherit Metab Dis 2008, Epub ahead of print.

50 Gassio R, Campistol J, Vilaseca MA, et al: Do adult patients with phenylketonuria improve their quality of life after introduction/resumption of a phenylalanine-restricted diet? Acta Paediatr 2003;92:1474-1478.

51 Waisbren SE, Hanley W, Levy HL, et al: Outcome at age 4 years in offspring of women with maternal phenylketonuria: the Maternal PKU Collaborative Study. JAMA 2000; 283:756-762.

52 Rohr F, Munier A, Sullivan D, et al: The Resource Mothers Study of Maternal Phenylketonuria: preliminary findings. J Inherit Metab Dis 2004;27:145-155.

53 Fiege B, Blau N: Assessment of tetrahydrobiopterin (BH4) responsiveness in phenylketonuria. J Pediatr 2007;150:627-630. 
-54 Trefz FK, Burton BK, Longo N, et al: Efficacy of sapropterin dihydrochloride in increasing phenylalanine tolerance in children with phenylketonuria: a phase III, randomized, double-blind, placebo-controlled study. J Pediatr 2009;154:700-707.

55 Burlina A, Blau N: Effect of BH(4) supplementation on phenylalanine tolerance. J Inherit Metab Dis 2009;32:40-45.

56 Hennermann JB, Buhrer C, Blau N, et al: Long-term treatment with tetrahydrobiopterin increases phenylalanine tolerance in children with severe phenotype of phenylketonuria. Mol Genet Metab 2005;86(suppl 1):S86-S90.

-57 Blau N, Belanger-Quintana A, Demirkol M, et al: Optimizing the use of sapropterin $\left(\mathrm{BH}_{4}\right)$ in the management of phenylketonuria. Mol Genet Metab 2009;96:158-163.

58 Singh R, Jurecki E, Rohr F: Recommendations for personalized dietary adjustments based on patient response to tetrahydrobiopterin $\left(\mathrm{BH}_{4}\right)$ in phenylketonuria. Top Clin Nutr 2008;23:149-157.

59 Broer S: Amino acid transport across mammalian intestinal and renal epithelia. Physiol Rev 2008;88:249-286.
60 Weglage J, Wiedermann D, Denecke Jet al: Individual blood-brain barrier phenylalanine transport determines clinical outcome in phenylketonuria. Ann Neurol 2001;50: 463-467.

61 Pietz J, Kreis R, Rupp A, et al: Large neutral amino acids block phenylalanine transport into brain tissue in patients with phenylketonuria. J Clin Invest 1999;103:1169-1178.

62 Schindeler S, Ghosh-Jerath S, Thompson S, et al: The effects of large neutral amino acid supplements in PKU: an MRS and neuropsychological study. Mol Genet Metab 2007;91: 48-54.

63 Matalon R, Michals-Matalon K, Bhatia G, et al: Double blind placebo control trial of large neutral amino acids in treatment of PKU: effect on blood phenylalanine. J Inherit Metab Dis 2007;30:153-158.

64 Ney DM, Hull AK, van Calcar SC, et al: Dietary glycomacropeptide supports growth and reduces the concentrations of phenylalanine in plasma and brain in a murine model of phenylketonuria. J Nutr 2008;138:316322.
65 Etzel MR: Manufacture and use of dairy protein fractions. J Nutr 2004;134:996S-1002S.

- 66 Veldhorst MA, Nieuwenhuizen AG, Hochstenbach-Waelen A, et al: Effects of complete whey-protein breakfasts versus whey without GMP-breakfasts on energy intake and satiety. Appetite 2009;52:388-395.

67 MacLeod EL, Clayton MK, van Calcar SC, et al: Breakfast with glycomacropeptide compared with amino acids suppresses plasma ghrelin levels in individuals with phenylketonuria. Mol Genet Metab 2010, E-pub ahead of print. DOI: 10.1016/j.ymgme.2010.04.003.

68 Ney DM, Gleason ST, van Calcar SC, et al: Nutritional management of PKU with glycomacropeptide from cheese whey. J Inherit Metab Dis 2009;32:32-39.

69 Sanjurjo P, Aldamiz L, Georgi G, et al: Dietary threonine reduces plasma phenylalanine levels in patients with hyperphenylalaninemia. J Pediatr Gastroenterol Nutr 2003; 36:23-26.

70 van Spronsen FJ, Burgard P: The truth of treating patients with phenylketonuria after childhood: the need for a new guideline. J Inherit Metab Dis 2008;31:673-679. 